\title{
ESSENTIAL DIMENSION LOWERING MAPPINGS HAVING DENSE DEFICIENCY SET
}

\author{
BY \\ MLADEN BESTVINA
}

\begin{abstract}
Two classes of surjective maps $f: S^{m} \rightarrow S^{n}$ that are one-to-one over the image of a dense set are constructed. We show that for $m, n \geq 3$ there is a monotone surjection $f: S^{m} \rightarrow S^{n}$ that is one-to-one over the image of a dense set; and for $3 \leq n \leq m \leq 2 n-3$, each element of $\pi_{m}\left(S^{n}\right)$ can be represented as a monotone surjection $f: S^{m} \rightarrow S^{n}$ that is one-to-one over the image of a dense set.
\end{abstract}

1. Introduction. The present paper should be considered as a continuation of the study of surjective maps between spheres that are "one-to-one over the image of a dense set". (A surjection $f: X \rightarrow Y$ is one-to-one over the image of a dense set if there exists a dense set $D \subseteq X$ such that for each $y \in f(D), \# f^{-1}(y)=1$; \# = cardinality.)

First inconceivable examples of such maps were constructed by J. J. Walsh [Wa 5]. Specifically, for any pair $n \geq 3, d \geq 2$ of integers, Walsh has built a monotone, surjective map $f: S^{n} \rightarrow S^{n}$ of degree $d$ that is one-to-one over the image of a dense set.

More recently, in [Be-Wa], it has been established that for any $m, n \geq 2$ there is a surjection $f: S^{m} \rightarrow S^{n}$ that is one-to-one over the image of a dense set. By construction this map is not monotone and factors through a 1-dimensional compactum, and hence it is null-homotopic; even more, it has no stable values. (A point $y \in Y$ is a stable value of a map $f: X \rightarrow Y$ between metric spaces if there exists an open cover $U$ of $Y$ so that for every $U$-approximation $f^{\prime}$ to $f, y$ is in the image of $f^{\prime}$.)

In this paper we show:

(a) For $m, n \geq 3$ there is a monotone surjection $f: S^{m} \rightarrow S^{n}$ that is one-to-one over the image of a dense set.

(b) For $3 \leq n \leq m \leq 2 n-3$ each element of $\pi_{m}\left(S^{n}\right)$ can be represented as a monotone surjection $f: S^{m} \rightarrow S^{n}$ that is one-to-one over the image of a dense set. In particular, if $3 \leq n \leq m \leq 2 n-3$ and $\pi_{m}\left(S^{n}\right) \neq 0$ (e.g., $\left.\pi_{n+1}\left(S^{n}\right)=Z_{2}\right)$, there is a monotone, essential map $f: S^{m} \rightarrow S^{n}$ that is one-to-one over the image of a dense set (and hence, all values of $f$ are stable).

The techniques used in the paper stem from D. Wilson [Wi 1, Wi 2] and J. J. Walsh [Wa 1-Wa 5]. Mappings are constructed by making use of "defining sequences". Although the necessary definitions are given, and in that respect the

Received by the editors August 1, 1983 and, in revised form, March 6, 1984.

1980 Mathematics Subject Classification. Primary 54C10; Secondary 55Q99.

Key words and phrases. One-to-one over the image of a dense set, monotone map, essential map, stable values.

(C) 1985 American Mathematical Society $0002-9947 / 85 \$ 1.00+\$ .25$ per page 
paper is self-contained, familiarity with $[$ Wa 5] is desirable. We follow the notation developed in that paper.

The author is obliged to J. J. Walsh for his immense willingness for endless conversations about the problem. Without his unreserved support, the preparation of this paper would be impossible.

2. Preliminaries. For any family $P$ of subsets of a set $X$, and for any $A \subseteq X$, we define

$$
\operatorname{St}(A, P)=\bigcup\{p \in P: p \cap A \neq \varnothing\} .
$$

Following [Wa 5], by a (stratified) partition on a closed PL $n$-manifold $N$ we mean a collection $P=\left\{p_{1}, \ldots, p_{k}\right\}$ of closed subsets of $N$ that cover $N$ with the following properties.

(P1) Each $p \in P$ is a PL $n$-submanifold (with boundary) of $N$.

(P2) If $p_{i(1)}, \ldots, p_{i(t)}$ are mutually distinct elements of $P$, then $p_{i(1)} \cap \cdots \cap p_{i(t)}$ is either empty or an $(n-t+1)$-dimensional PL submanifold of the boundary of $p_{i(1)} \cap \cdots \cap p_{i(t-1)}$.

Observe that $p_{i(1)} \cap \cdots \cap p_{i(t)} \neq \varnothing$ has empty boundary if and only if $p \cap p_{i(1)} \cap$ $\cdots \cap p_{i(t)}=\varnothing$ for all $p \in P-\left\{p_{i(1)}, \ldots, p_{i(t)}\right\}$.

If $L$ is any triangulation of $N$, by $J_{i}^{N}$ denote the standard handlebody decomposition of $N$ associated with the $i$ th barycentric subdivision $\beta^{i} L$ of $L$ :

$$
J_{i}^{N}=\left\{\operatorname{St}\left(v, \beta^{i+1} L\right): v \text { is a vertex of } \beta^{i} L\right\} .
$$

It is easy to see that $J_{i}^{N}$ satisfies (P1) and (P2). For $i \geq 1$ and $j=\operatorname{St}\left(v, \beta^{i+1} L\right) \in$ $J_{i}^{N}$, define the index of $j, \operatorname{Ind}(j)$, to be equal to $k$ if $v$ is the barycenter of a $k$-simplex in $\beta^{i} L$.

Let $M^{m}, N^{n}$ be PL manifolds, and $P, Q$ partitions on $M, N$ respectively. We say that a function $T: P \rightarrow Q$ is admissible, provided:

(A1) $T$ is a bijection;

(A2) for all $p_{i(1)}, \ldots, p_{i(t)} \in P$,

$$
p_{i(1)} \cap \cdots \cap p_{i(t)} \neq \varnothing \Rightarrow T\left(p_{i(1)}\right) \cap \cdots \cap T\left(p_{i(t)}\right) \neq \varnothing
$$

(A3) for all $p, p^{\prime} \in P$,

$$
p \cap p^{\prime} \neq \varnothing \Rightarrow T(p) \cap T\left(p^{\prime}\right) \neq \varnothing .
$$

Let $L$ be any triangulation of $N$. If $T: P \rightarrow J$ is a triple satisfying (A2) and (A3), where $J=J_{i}^{N}$ is the handlebody decomposition of $N$ associated with $\beta^{i} L$, by an induced map we mean any map $h: M \rightarrow N$ with $h(p) \subseteq T(p)$ for all $p \in P$. We can define $h$ by the "backward induction" on $t$, requiring that $h\left(p_{i(1)} \cap \cdots \cap p_{i(t)}\right) \subseteq$ $T\left(p_{i(1)}\right) \cap \cdots \cap T\left(p_{i(t)}\right)$. Since each nonempty intersection of elements of $J$ is an absolute retract, the inductive step "goes through". The same fact establishes that any two induced maps are homotopic (see [Wa 5]), which enables us to talk about the induced map.

A sequence of triples $\left\{T_{i}: P_{i} \rightarrow J_{i}\right\}_{i=0}^{\infty}$ is a defining sequence provided, for all $i \geq 0$ :

(DS1) $J_{i}=J_{i}^{N}$ is the handlebody decomposition of $N$ associated with $\beta^{i} L$;

(DS2) $P_{i}$ is a partition on $M$;

(DS3) $T_{i}$ is an admissible function; 
(DS4) for all $p \in P_{i}, p^{\prime} \in P_{i+1}$,

$$
p \cap p^{\prime} \neq \varnothing \Leftrightarrow \operatorname{Int}\left(p \cap p^{\prime}\right) \neq \varnothing \Leftrightarrow T_{i}(p) \cap T_{i+1}\left(p^{\prime}\right) \neq \varnothing .
$$

The reader should find establishing the following result a useful exercise.

PROPOSITION 2.1 (SEE [Wa 5]). Let $\left\{T_{i}: P_{i} \rightarrow J_{i}\right\}_{i=0}^{\infty}$ be a defining sequence.

(i) Setting $h^{-1}(y)=\bigcap_{i=0}^{\infty} \operatorname{St}\left(p_{i}, P_{i}\right)$ for any choice of $p_{i} \in P_{i}$ with $T_{i}\left(p_{i}\right) \ni y$ defines a surjective map $h: M \rightarrow N$. Moreover, $\operatorname{Int} \operatorname{St}\left(p_{i}, P_{i}\right) \supseteq \operatorname{St}\left(p_{i+1}, P_{i+1}\right)$ $(i=0,1,2, \ldots)$.

(ii) If $h_{i}: M \rightarrow N$ is the map induced by $T_{i}: P_{i} \rightarrow J_{i}$, then $h=\lim _{i \rightarrow \infty} h_{i}$ and $h_{0} \simeq h_{1} \simeq h_{2} \simeq \cdots \simeq h$.

(iii) If each $p \in P_{i}, i \geq 0$, is connected, then $h$ is a monotone map.

(iv) If for each $i \geq 1$ and each $j \in J_{i}$ with $\operatorname{Ind}(j)=n$ there exists a $P L$ m-cell $B \subseteq M$ with

$$
\operatorname{St}\left(T_{i}^{-1}(j), P_{i+1}\right) \subseteq \operatorname{Int} B \subseteq \operatorname{St}\left(T_{i}^{-1}(j), P_{i}\right),
$$

then the points $y \in N$ for which $h^{-1}(y) \subseteq M$ is a cellular set form a dense subset of $N$.

To construct interesting maps between manifolds using 2.1 , we have to produce defining sequences. The major step consists of generating a triple $T_{i+1}: P_{i+1} \rightarrow$ $J_{i+1}$ from a triple $T_{i}: P_{i} \rightarrow J_{i}$ previously constructed. To make the notation easier, the triple $T_{i}: P_{i} \rightarrow J_{i}$ will be denoted by $T: P \rightarrow J$, and the triple $T_{i+1}: P_{i+1} \rightarrow$ $J_{i+1}$ by $\tilde{T}: \tilde{P} \rightarrow \tilde{J}$. Coherently, we will rename the subdivision $\beta^{i} L$ and again call it $L$. Hence

$$
\begin{aligned}
& J=\{\operatorname{St}(v, \beta L): v \text { is a vertex of } L\}, \\
& \tilde{J}=\left\{\operatorname{St}\left(v, \beta^{2} L\right): v \text { is a vertex of } \beta L\right\} .
\end{aligned}
$$

The construction of $\tilde{P}$ is in two stages. We define an intermediate triple $\hat{T}: \hat{P} \rightarrow$ $\tilde{J}$. Warning. $\hat{P}$ will be a partition of $M$, and $\hat{T}$ will satisfy (A2) and (A3), but not necessarily (A1).

The elements of $\hat{P}$ will be indexed by the set $S$ of all collections $\left\{p_{i(1)}, \ldots, p_{i(t)}\right\}$ $\subseteq P$ that have nonempty intersections. (These intersections, in Walsh's terminology, are called the strata of $P$.)

The collection $\hat{P}=\left\{p_{s}, s \in S\right\}$ will satisfy the following properties.

(H1) $\hat{P}$ is a partition on $M$.

(H2) $p_{s(1)}, \ldots, p_{s(t)} \in \hat{P}$ have nonempty intersection if and only if $\{s(1), \ldots, s(t)\}$ $\subseteq S$ is well-ordered with respect to inclusion.

(H3) For any $p \in P$ and $p_{s} \in \hat{P}$,

$$
p \cap p_{s} \neq \varnothing \Leftrightarrow \operatorname{Int}\left(p \cap p_{s}\right) \neq \varnothing \Leftrightarrow p \in s .
$$

(H4) For any $p \in P, p$ and $p_{\{p\}}$ are homeomorphic.

Still following [Wa 5], we construct the elements $p_{s} \in \hat{P}$ as follows (see Figure 1). Let $K$ be a triangulation of $M$ so that each stratum $\bigcap s, s \in S$, is a full subcomplex of $K$. Define the core of $s \in S$ by

$$
c(s)=\bigcup\{\tau: \tau \text { is a simplex of } \beta K \text { contained in } \bigcap s-\partial(\bigcap s)\} .
$$

Finally, set

$$
p_{s}=\bigcup\left\{\operatorname{St}\left(v, \beta^{2} K\right): v \in c(s) \text { is a vertex of } \beta K\right\}
$$


Observe that, by choosing a sufficiently small triangulation $K$ :

(H5) Given neighborhoods $U(p)$ of $p \in P$, we can arrange that $\operatorname{St}(p, \hat{P}) \subseteq U(p)$ for all $p \in P$.

We can also define the function $\hat{T}: \hat{P} \rightarrow \tilde{J}$ by $\hat{T}\left(p_{s}\right)=\operatorname{St}\left(v, \beta^{2} L\right) \in \tilde{J}$, where $v$ is determined as follows. If $s=\left\{p_{i(1)}, \ldots, p_{i(t)}\right\}$ and $T\left(p_{i(r)}\right)=\operatorname{St}\left(v_{r}, \beta L\right)$, then $v$ is the barycenter of the simplex whose vertices are $v_{1}, \ldots, v_{t}$. Property (H2) implies that $\hat{T}$ satisfies (A2) and (A3). It is evident that $\hat{T}$ is a one-to-one function (but not necessarily a surjection).

We will "repair" the triple $\hat{T}: \hat{P} \rightarrow \tilde{J}$ to get the triple $\tilde{T}: \tilde{P} \rightarrow \tilde{J}$, but the reparation will depend on the desired properties of the function $h: M \rightarrow N$ determine by the defining sequence. The "reparation process", as well as the construction of the triple $T_{0}: P_{0} \rightarrow J_{0}$, is explained in detail in forthcoming sections.

REMARK. If the partiion $P$ is the standard handlebody decomposition corresponding to a triangulation $K$ of the manifold $M$, then the partition $\hat{P}$ constructed above is (up to an ambient isotopy) the standard handlebody decomposition of $M$ corresponding to the barycentric subdivision $K^{\prime}$ of $K$. This fact will be implicitly used in the sequel.

3. Essential maps. The purpose of this section is to establish the following

Proposition 3.1. Let $f: S^{m} \rightarrow S^{n}$ be any map, and let $3 \leq n \leq m \leq 2 n-3$. Then there exists a surjective monotone map $h: S^{m} \rightarrow S^{n}$ homotopic to $f$ such that the set $\left\{y \in S^{n}: h^{-1}(y)\right.$ is cellular in $\left.S^{m}\right\}$ is dense in $S^{n}$.

A routine consequence of 3.1 is the result announced in the Introduction.

THEOREM 3.2. For any map $f: S^{m} \rightarrow S^{n}, 3 \leq n \leq m \leq 2 n-3$, there exists a surjective monotone map $g: S^{m} \rightarrow S^{n}$ homotopic to $f$ that is one-to-one over the image of a dense set.

Proof. Let $h: S^{m} \rightarrow S^{n}$ be a map whose existence is promised by 3.1 . We "carefully shrink countably many cellular fibers of $h$ " in order to obtain the soughtafter map $g: S^{m} \rightarrow S^{n}$. The shrinking process can be described as follows.

Let $U_{1}, U_{2}, \ldots$ be a countable basis of open sets of $S^{m}$. Choose a fiber $F$ of $h$ with $F \cap U_{1} \neq \varnothing$, and pick a cellular fiber $C$ of $h$ in a "small" connected neighborhood $V$ of $F$ (here we use the fact that $h$ is a monotone map). Let $\lambda: S^{m} \rightarrow S^{m}$ be a surjection whose only nondegenerate point-preimage is $C$. We can arrange that $\lambda(C) \in U_{1}$ and $\lambda=$ identity off of $V$. Then $g_{1}=h \lambda^{-1}$ is a monotone surjection "close" to $h$, and one of the fibers of $g_{1}$ is a point in $U_{1}$. In a similar fashion we produce monotone maps $g_{2}, g_{3}, \ldots$ such that $g_{i+1}$ is "close" to $g_{i}$, it agrees with $g_{i}$ off of a "small" neighborhood of a fiber of $g_{i}$, and $g_{i+1}$ has degenerate point-preimages in each of the sets $U_{1}, \ldots, U_{i+1}$.

Exercising sufficient control on all choices made, and carefully interpreting the quoted words in the preceding paragraph, we can arrange that the sequence $g_{1}$, $g_{2}, \ldots$ converges to a monotone map $g: S^{m} \rightarrow S^{n}$ homotopic to $h$ that is one-toone over the image of a dense set.

Before giving a proof of Proposition 3.1, we state and prove an interesting corollary of Theorem 3.2. 
In what follows, $E^{r}$ denotes Euclidean $r$-dimensional space. Observe that the homogeneity properties of $E^{r}$ establish that the set of all stable values of a surjection $f: X \rightarrow E^{r}$ is open in $E^{r}$.

COROLLARY 3.3. Let $m, n \geq 2$ be integers.

(i) If $\pi_{m-1}\left(S^{n-1}\right)=0$, then any surjection $f: E^{m} \rightarrow E^{n}$ that is one-to-one over the image of a dense set has no stable values.

(ii) If $3 \leq n \leq m \leq 2 n-3$ and $\pi_{m-1}\left(S^{n-1}\right) \neq 0$, then there exists a proper monotone surjection $f: E^{m} \rightarrow E^{n}$ that is one-to-one over the image of a dense set and has all values stable.

PROOF. (i) In view of the observation made before the statement of Corollary 3.3 , it suffices to prove that if $\# f^{-1}(y)=1$, then $y$ is not a stable value of $f$. Let $B$ be a "small" ball around $f^{-1}(y)$. The assumption about the homotopy group reveals that $f \mid \partial B$ is a null-homotopic map in a "small" deleted neighborhood of $y$. Redefine $f$ in Int $B$, using the homotopy, to get an approximation $f^{\prime}$ to $f$ whose image misses $y$.

(ii) By Freudenthal's Suspension Theorem (see [Sp, p. 458]), $\pi_{m-1}\left(S^{n-1}\right) \cong$ $\pi_{m}\left(S^{n}\right)$. Application of 3.2 gives an essential monotone surjection $f: S^{m} \rightarrow S^{n}$ that is one-to-one over the image of a dense set. Pick $y \in S^{n}$ such that $\# f^{-1}(y)=1$. Then $f /: S^{m}-f^{-1}(y) \rightarrow S^{n}-y$ is a proper monotone surjection that is one-to-one over the image of a dense set. All values of $f /$ are stable since the opposite would violate the fact that $f$ is essential.

PROOF OF 3.1. We construct a defining sequence $\left\{T_{i}: P_{i} \rightarrow J_{i}\right\}_{i=0}^{\infty}$ with the following additional properties.

(E1) For mutually distinct elements $p_{i(1)}, \ldots, p_{i(t)} \in P_{i}$,

$$
p_{i(1)} \cap \cdots \cap p_{i(t)} \neq \varnothing \Leftrightarrow t \leq n+1 \text { and } T\left(p_{i(1)} \cap \cdots \cap T\left(p_{i(t)}\right)\right) \neq \varnothing
$$

$(i=0,1, \ldots)$.

(E2) Each element $p \in P_{i}$ is $(m-n)$-connected $(i=0,1, \ldots)$.

(E3) If $j \in J_{i}, \operatorname{Ind}(j)=n$, then $T_{i}^{-1}(j)$ is a PL $m$-ball $(i=1,2, \ldots)$.

As announced in $\S 2$, we construct the defining sequence by induction. Suppressing indices, we start with an admissible function $T: P \rightarrow J$ satisfying (E1) and (E2) (produced following the inductive analysis). Let $K$ be a triangulation of $S^{m}$ such that all strata of $P$ are full subcomplexes of $K$. Let $\hat{T}: \hat{P} \rightarrow \tilde{J}$ be the triple constructed in $\S 2, \hat{P}=\left\{p_{s}, s \in S\right\}$. Observe that (E1) implies that $\hat{T}$ satisfies (A1)-(A3). Also, (H2) implies that $\hat{T}$ satisfies (E1).

We now "repair" the triple $\hat{T}: \hat{P} \rightarrow \tilde{J}$ to get another triple $\tilde{T}: \tilde{P} \rightarrow \tilde{J}$ which satisfies (E2) and (E3). We want to maintain all properties that $\hat{T}: \hat{P} \rightarrow \tilde{J}$ already satisfies. For all $s \in S$ choose $p(s) \in s$; if possible, choose $p(s)$ so that Ind $T(p(s))=$ $n$. A quick remark: each $s \in S$ contains at most one $p$ with $\operatorname{Ind} T(p)=n$. We interrupt the proof to introduce some notation.

For a compactum $X$, denote by $C(X)=X \times[0,1] /\left(x_{1}, 1\right) \sim\left(x_{2}, 1\right)$ the cone over $X$. We identify $X=X \times\{0\} \subseteq C(X)$. Name $\frac{1}{2} C(X)=X \times\left[0, \frac{1}{2}\right] \subset C(X)$ the bottom half of the cone over $X$. If $A$ is a subcomplex of $K$, by $A^{(r)}$ we denote the $r$-skeleton of $A$ with respect to $K$. Finally, " $\approx$ " means "PL homeomorphic".

For all $s \in S$ with \#s>1 choose a polyhedron $X_{s} \subseteq S^{m}$ containing $c(s)$ with the following properties. 
(a) $X_{s} \subseteq p(s) \cap\left(p_{s} \cup \operatorname{Int} p_{\{p(s)\}}\right)$;

(b) $X_{s} \cap \partial p(s)=c(s)$;

(c) $\left(X_{s}, X_{s} \cap p_{s}, c(s)\right) \approx\left((c(s)) \cup C\left((c(s))^{(m-n)}\right), c(s) \cup \frac{1}{2} C\left((c(s))^{(m-n)}\right), c(s)\right)$, and

(d) if $s_{1} \neq s_{2}$, then $X_{s_{1}} \cap X_{s_{2}}=\varnothing$.

Sets $X_{s}$ exist, since $\operatorname{dim} C\left(c(s)^{(m-n)}\right) \leq m-n+1$, and $2(m-n+1)<m$. The same inequality, coupled with (c) and the fact that each $p_{s}$ with \#s = 1 is $(m-n)$-connected (see (H4)), testifies that $p_{\{p(s)\}} \backslash \bigcup\left\{X_{s^{\prime}}: s^{\prime} \in S, \# s^{\prime}>1\right\}$ is still $(m-n)$-connected for all $s \in S$.

Let $K^{\prime}$ be a subdivision of $K$ such that all mentioned subsets of $S^{m}$ are full subcomplexes with respect to $K^{\prime}$. Let $N_{s}$ be the second derived neighborhood of $X_{s}$ in $p(s)$ with respect to $K^{\prime}$. Define

$$
\tilde{p}_{s}=\left\{\begin{array}{l}
p_{s} \cup N_{s} \quad \text { if } \# s>1, \\
p_{s} \backslash \bigcup\left\{\operatorname{Int} N_{s^{\prime}}, s^{\prime} \in S, \# s^{\prime}>1\right\} \quad \text { if } \# s=1
\end{array}\right.
$$

The reader can easily verify that $\tilde{P}=\left\{\tilde{p}_{s}, s \in S\right\}$ is a partition on $S^{m}$, that $\tilde{T}: \tilde{P} \rightarrow \tilde{J}$ defined by $\tilde{T}\left(\tilde{p}_{s}\right)=\hat{T}\left(p_{s}\right)$ is an admissible function, and that the triple $\tilde{T}: \tilde{P} \rightarrow \tilde{J}$ satisfies (E1) and (E2). (Note that $p_{s}$ collapses to $c(s)$; by adding the cone over $c(s)^{(m-n)}$, we "killed" first $(m-n)$ homotopy groups).

If $\# s=n+1$, then $\tilde{p}_{s}$ is a regular neighborhood of $X_{s} \approx C(c(s)$ ) (since $\operatorname{dim}(c(s))=m-n)$, and hence $\tilde{p}_{s}$ is an $m$-ball. This establishes (E3). From (a) and (H3), it easily follows that $T$ and $\tilde{T}$ satisfy (DS4).

Observe that, by our choice of $p(s), s \in S$, we have $\operatorname{St}(p, \tilde{P})=\operatorname{St}(p, \hat{P})$, for all $p \in P$ with Ind $T(p)=n$. Thus, taking into account (H5), we get:

(E4) Given neighborhoods $U(p)$ of $p \in P$ with $\operatorname{Ind} T(p)=n$, we can arrange that $\operatorname{St}(p, \tilde{P}) \subseteq U(p)$ for all such $p$.

If $h: S^{m} \rightarrow S^{n}$ is the map associated with a defining sequence $\left\{T_{i}: P_{i} \rightarrow J_{i}\right\}_{i=0}^{\infty}$ satisfying (E1)-(E3), then, by 2.1 (iii), $h$ is a monotone surjection, and using 2.1 (iv), together with (E4), we see that we can arrange that the set $\left\{y \in S^{n}: h^{-1}(y)\right.$ is cellular $\}$ is dense in $S^{n}$. Indeed, let $B_{j}$ be a regular neighborhood of $T_{i}^{-1}(j)$ contained in $\operatorname{St}\left(T_{i}^{-1}(j), P_{i}\right)$. By (E3), $B_{j}$ is an $m$-ball. By (E4) we can arrange that $\operatorname{St}\left(T_{i}^{-1}(j), P_{i+1}\right) \subseteq \operatorname{Int} B_{j}$.

To finish the proof of 3.1 , in view of 2.1 (ii), we need to construct a triple $T_{0}: P_{0} \rightarrow J_{0}$ satisfying (E1) and (E2) such that the induced map $h_{0}: S^{m} \rightarrow S^{n}$ is homotopic to $f$. By Freudenthal's Suspension Theorem [Sp, p. 458], there is a map $f^{\prime}: S^{m-1} \rightarrow S^{n-1}$ whose suspension $\Sigma f^{\prime}: S^{m} \rightarrow S^{n}$ is homotopic to $f$. Without loss of generality, we may assume that $f^{\prime}$ is a surjective simplicial map, with respect to some triangulations $K_{0}, L_{0}$ of $S^{m-1}, S^{n-1}$ respectively. Then the map $\Sigma f^{\prime}: \Sigma K_{0} \rightarrow \Sigma L_{0}$ is simplicial. To suppress unnecessary symbols, rename it as $f: K \rightarrow L$. Let

$$
p_{v}=\bigcup\{\operatorname{St}(w, \beta K): f(w)=v, w \text { is a vertex of } K\}
$$

and set $P=\left\{p_{v}: v\right.$ is a vertex of $\left.L\right\}$. Then $P$ is a partition on $S^{m}$, and the function $T: P \rightarrow J_{0}$ given by $T\left(p_{v}\right)=\operatorname{St}(v, \beta L)$ satisfies (A1)-(A3) and (E1).

We now "repair" the triple $T: P \rightarrow J_{0}$ to get a new triple $T_{0}: P_{0} \rightarrow J_{0}$ satisfying, in addition, (E2). The strategy is the same as for obtaining $\tilde{T}$ from $\hat{T}$. Observe 
that if $\sigma$ is a suspension vertex of $L$, then $p_{\sigma}$ is an $m$-ball. Moreover, $p_{\sigma}$ intersects all elements of $P$ except for $p_{\tau} \in P$, where $\tau$ is the other suspension vertex. If $p \in P-\left\{p_{\sigma}, p_{\tau}\right\}$, then $p$ collapses to $p \cap p_{\sigma}$. For each $p \in P-\left\{p_{\sigma}, p_{\tau}\right\}$ choose a polyhedron $A_{p} \subseteq p \cap p_{\sigma}$ such that $\operatorname{dim} A_{p} \leq m-n$ and the pair $\left(p \cap p_{\sigma}, A_{p}\right)$ is $(m-n)$-connected. We can arrange that $A_{p(1)} \cap A_{p(2)}=\varnothing$ if $p(1) \neq p(2)$. (We can take $A_{p}$ to be the $(m-n)$-skeleton of a shrunk copy of $p \cap p_{\sigma}$.) Next, embed the cones $C\left(A_{p}\right)$ into $p_{\sigma}$ to obtain polyhedra $Y(p), p \in P-\left\{p_{\sigma}, p_{\tau}\right\}$. We can arrange that $Y(p) \cap \partial p_{\sigma}=A_{p}$ for all $p \in P-\left\{p_{\sigma}, p_{\tau}\right\}$ and, by general positioning, that $Y(p) \cap Y\left(p^{\prime}\right)=\varnothing$ for $p \neq p^{\prime}$ (we are in the range of dimensions where $2(m-n+1)<$ $m)$. Let $K^{\prime}$ be a subdivision of $K$ such that all mentioned subsets of $S^{m}$ are full subcomplexes of $K^{\prime}$. If $N_{v}$ is the second derived neighborhood of $Y\left(P_{v}\right)$ in $p_{\sigma}$, set

$$
\tilde{p}_{v}=\left\{\begin{array}{l}
p_{v} \cup N_{v}, \quad v \text { is a vertex of } K_{0}, \\
p_{\sigma}-\bigcup\left\{\text { Int } p_{v^{\prime}}, v^{\prime} \text { is a vertex of } K_{0}\right\}, \quad v=\sigma, \\
p_{\tau}, \quad v=\tau .
\end{array}\right.
$$

Then $P_{0}=\left\{\tilde{p}_{v}: v\right.$ is a vertex of $\left.K\right\}$ is a partition on $S^{m}$, and $T_{0}: P_{0} \rightarrow J_{0}$ defined by $T_{0}\left(\tilde{p}_{v}\right)=T\left(p_{v}\right)$ satisfies (A1)-(A3), (E1) and (E2). If $h_{0}: S^{m} \rightarrow S^{n}$ is a map induced by $T_{0}: P_{0} \rightarrow J_{0}$, then $h_{0}$ and $f$ are $\mathcal{U}$-close, where $\mathcal{U}=\left\{\operatorname{St}\left(j, J_{0}\right), j \in J_{0}\right\}$ is a closed cover of $S^{n}$ such that each nonempty intersection of elements of $\mathcal{U}$ is an absolute retract (in fact, it is a PL ball). Hence (see [Wa 5]) $h_{0}$ and $f$ are homotopic maps.

This finishes the proof of 3.1 .

4. Monotone maps. In $\S 3$ we have shown that, in certain range of dimensions, there exist essential, monotone maps $f: S^{m} \rightarrow S^{n}$ that are one-to-one over the image of a dense set. In this section we show how to construct monotone (inessential) surjections $f: S^{m} \rightarrow S^{n}$ that are one-to-one over the image of a dense set for any $m, n \geq 3$. If $m>n \geq 4$, the existence of such maps follows from 3.2. Indeed, let $f_{i}: S^{i} \rightarrow S^{i-1}$ be a monotone surjection that is one-to-one over the image of a dense set $(i=n+1, n+2, \ldots, m)$. Let $g_{i}: S^{i} \rightarrow S^{i}$ be a homeomorphism intermingling the two pertinent (countable) dense subsets of $S^{i}(i=$ $n+1, n+2, \ldots, m-1)$. Then the composition $f_{n+1} g_{n+1} \cdots f_{m-1} g_{m-1} f_{m}: S^{m} \rightarrow$ $S^{n}$ is a map with the desired properties. However, we want to present an independent proof that also works for $3 \leq m \leq n$ or $n=3$.

THEOREM 4.1. For any $m, n \geq 3$ there exists a monotone surjection $h: S^{m} \rightarrow$ $S^{n}$ that is one-to-one over the image of a dense set.

In the proof we need

LEMMA 4.2. Let $h: X \rightarrow Y$ be a surjective map between compact metric spaces. If each nonempty open set in $X$ contains a fiber of $h$, then $h$ is one-to-one over the image of a dense set.

ProOF. Suppose not. Let $F_{\varepsilon}=\bigcup\left\{h^{-1}(y): y \in Y\right.$, diam $\left.h^{-1}(y) \geq \varepsilon\right\}$. Then $F_{\varepsilon}$ is a closed set for any $\varepsilon>0$, and $\bigcup\left\{F_{\varepsilon}, \varepsilon>0\right\}$ has nonempty interior. By Baire's Category Theorem [Du, p. 250], there exists $\varepsilon>0$ such that $F_{\varepsilon}$ has nonempty interior. Let $U \subseteq F_{\varepsilon}$ be a nonempty open set with $\operatorname{diam} U<\varepsilon$. Then $U$ does not contain any fibers of $h$, contrary to the hypothesis. 
ProOF OF 4.1. We construct a defining sequence $\left\{T_{i}: P_{i} \rightarrow J_{i}\right\}_{i=0}^{\infty}$ with the following properties.

(M1) Each $p \in P_{i}$ is connected, $i=0,1,2, \ldots$

(M2) If $p_{i(1)}, p_{i(2)}, p_{i(3)}, p_{i(4)} \in P_{i}$ are mutually distinct elements, then $p_{i(1)} \cap$ $p_{i(2)} \cap p_{i(3)} \cap p_{i(4)}=\varnothing$.

As in $\S 3$, we show first how to construct the triple $T_{i+1}: P_{i+1} \rightarrow J_{i+1}$ from the triple $T_{i}: P_{i} \rightarrow J_{i}$ already constructed. As before, we suppress indices, starting with an admissible function $T: P \rightarrow J$ satisfying (M1) and (M2). Let $\hat{T}: \hat{P} \rightarrow \tilde{J}$ be the triple constructed in $\S 2$. Observe that, although it is one-to-one, $T$ will never be onto (this is the whole point of the construction).

We have to create new elements of $P$ that will correspond to elements of $\tilde{J}-\operatorname{Im} \hat{T}$, as well as make all elements of $\hat{P}$ connected. Observe that if $j \in \tilde{J}$ with $\operatorname{Ind}(j) \leq 1$, then (by (A3)) $j \in \operatorname{Im} \hat{T}$; and if $\operatorname{Ind}(j) \geq 3$, then (by (M2)) $j \notin \operatorname{Im} \hat{T}$.

First "connect up" all components of elements of $\hat{P}$. The only important property of $\hat{P}$ we use here is that for each disconnected $p_{s} \in \hat{P}$ there is a connected element $\hat{p} \in \hat{P}$ such that each component of $p_{s}$ intersects $\hat{p}$. (If $p \in s, \hat{p}=p_{\{p\}}$ would do; see (H4).) Fixing a triangulation $K^{\prime}$ of $S^{m}$ such that all pertinent subsets of $S^{m}$ are (full) subcomplexes, for each disconnected $p \in \hat{P}$ choose a PL arc $\alpha_{p}$ lying in a connected element $c(p) \in \hat{P}$ such that $\alpha_{p} \cap \partial c(p)$ is a finite set intersecting each component of $p$. We can also arrange that different arcs are disjoint, and lie in the complement of the $(m-2)$-skeleton of $K^{\prime}$ (here we use $m \geq 3$ ). Choose a subdivision $K^{\prime \prime}$ of $K^{\prime}$ such that $\alpha_{p}$ 's are subcomplexes with respect to $K^{\prime \prime}$, and let $N_{p}$ be the second derived neighborhood of $\alpha_{p}$ in $c(p)$. Finally, set

$$
p^{0}=\left\{\begin{array}{l}
p \cup N_{p} \text { if } p \in \hat{P} \text { is disconnected, } \\
p-\bigcup\left\{N_{p^{\prime}}: p^{\prime} \in \hat{P} \text { is disconnected }\right\} \quad \text { if } p \in \hat{P} \text { is connected. }
\end{array}\right.
$$

Set $\hat{P}^{0}=\left\{p^{0}, p \in \hat{P}\right\}$. Then $\hat{T}^{0}: \hat{P}^{0} \rightarrow \tilde{J}$ defined by $\hat{T}^{0}\left(p_{s}^{0}\right)=\hat{T}\left(p_{s}\right)$ is a triple satisfying (A2), (A3), (M1) and (M2).

Now, we create new elements so that $\hat{T}^{0}$ can be extended to a bijection.

Let $\tilde{J}-\operatorname{Im} \hat{T}^{0}=\left\{j_{1}, j_{2}, \ldots, j_{r}\right\}$. We can order this set so that $k \leq l$ implies $\operatorname{Ind}\left(j_{k}\right) \leq \operatorname{Ind}\left(j_{l}\right)$. We define a sequence $\left\{\hat{T}^{k}: \hat{P}^{k} \rightarrow \tilde{J}\right\}_{k=1}^{r}$ of triples satisfying (A2), (A3), (M1), (M2) and

$\left(\mathrm{a}_{k}\right) \tilde{J}-\operatorname{Im} \hat{T}^{k}=\left\{j_{k+1}, j_{k+2}, \ldots, j_{r}\right\}$

$\left(\mathrm{b}_{k}\right)$ for any $p \in P, p^{\prime} \in \hat{P}^{k}$,

$$
p \cap p^{\prime} \neq \varnothing \Leftrightarrow \operatorname{Int}\left(p \cap p^{\prime}\right) \neq \varnothing \Leftrightarrow T(p) \cap \hat{T}^{k}\left(p^{\prime}\right) \neq \varnothing .
$$

Clearly, $\hat{T}^{0}: \hat{P}^{0} \rightarrow \tilde{J}$ satisfies $\left(\mathrm{a}_{0}\right)$ and $\left(\mathrm{b}_{0}\right)$. Finally, we set $\{\tilde{T}: \tilde{P} \rightarrow \tilde{J}\}=$ $\left\{\hat{T}^{r}: \hat{P}^{r} \rightarrow \tilde{J}\right\}$.

An argument for the inductive step is as follows. Assume the triple $\hat{T}^{k-1}: \hat{P}^{k-1}$ $\rightarrow \tilde{J}$ satisfies (A2), (A3), (M1), (M2), ( $\left.\mathrm{a}_{k-1}\right)$ and $\left(\mathrm{b}_{k-1}\right)$. Define

$$
B=\bigcup\left\{p \in \hat{P}^{k-1}: \hat{T}^{k-1}(p) \cap j_{k} \neq \varnothing\right\}
$$

Claim 1. $B$ is connected.

Indeed, let $p_{1}, p_{2} \in \hat{P}^{k-1}$ with $\hat{T}^{k-1}\left(p_{i}\right) \cap j_{k} \neq \varnothing, i=1,2$. Let $v_{1}, v_{2}$ be the two vertices of $\beta L$ such that $\hat{T}^{k-1}\left(p_{i}\right)=\operatorname{St}\left(v_{i}, \beta^{2} L\right), i=1,2$. Similarly, let $v$ be 
the vertex of $\beta L$, such that $j_{k}=\operatorname{St}\left(v, \beta^{2} L\right)$. Let $\sigma, \sigma_{1}, \sigma_{2}$ be the simplexes of $L$ whose barycenters are $v, v_{1}, v_{2}$ respectively. Since $\operatorname{St}\left(v, \beta^{2} L\right) \cap \operatorname{St}\left(v_{i}, \beta^{2} L\right) \neq \varnothing, \sigma$ and $\sigma_{i}$ are comparable simplexes, $i=1,2$ (i.e. one is a face of the other). Choose a sequence $w_{1}, w_{2}, \ldots, w_{l}$ of vertices of $\sigma$, such that $w_{1}$ is a vertex of $\sigma_{1}$, and $w_{l}$ is a vertex of $\sigma_{2}$. Say, $\sigma_{1}=\left\langle w_{1}, a_{1}, \ldots, a_{q}\right\rangle, \sigma_{2}=\left\langle w_{l}, b_{1}, \ldots, b_{u}\right\rangle$. In the sequence $\left\langle w_{1}, a_{1}, \ldots, a_{q-1}, a_{q}\right\rangle,\left\langle w_{1}, a_{1}, \ldots, a_{q-1}\right\rangle, \ldots,\left\langle w_{1}, a_{1}\right\rangle,\left\langle w_{1}\right\rangle,\left\langle w_{1}, w_{2}\right\rangle,\left\langle w_{2}\right\rangle,\left\langle w_{2}, w_{3}\right\rangle$, $\left\langle w_{3}\right\rangle, \ldots,\left\langle w_{l-1}, w_{l}\right\rangle,\left\langle w_{l}\right\rangle,\left\langle w_{l}, b_{1}\right\rangle, \ldots,\left\langle w_{l}, b_{1}, \ldots, b_{u}\right\rangle$ any two consecutive simplexes of $L$ are comparable. Let $j^{1}, j^{2}, \ldots, j^{x}$ be the corresponding sequence of elements of $J$ (determined by the barycenters of the simplexes in the sequence). Note that (1) any two consecutive elements in this sequence intersect, (2) we can arrange the vertices of $\sigma_{1}$ and $\sigma_{2}$ so that each element in the sequence intersects $j_{k}$, and (3) by our choice of indexing elements of $J-\operatorname{Im} \hat{T}^{0}$ and the fact that $\operatorname{Ind}(j) \leq 1$ implies $j \in \operatorname{Im} T^{0}$, each element in the sequence is in $\operatorname{Im} \hat{T}^{k-1}$. Now by (A3) and (M1), $\left(\hat{T}^{k-1}\right)^{-1}\left(j^{1}\right),\left(\hat{T}^{k-1}\right)^{-1}\left(j^{2}\right), \ldots,\left(\hat{T}^{k-1}\right)^{-1}\left(j^{x}\right)$ is a sequence of connected sets whose union contains $p_{1}, p_{2}$ and itself is contained in $B$ such that any two consecutive elements intersect. Hence, $B$ is connected.

Claim 2. If $p \in P$ and if $T(p) \cap j_{k} \neq \varnothing$, then $\operatorname{Int}(B \cap p) \neq \varnothing$.

Indeed, if $j_{k}=\operatorname{St}\left(v, \beta^{2} L\right)$, and if $v$ is a barycenter of a simplex $\sigma=\left\langle a_{1}, \ldots, a_{l}\right\rangle$ of $L$, then $T(p)=\operatorname{St}\left(a_{i}, \beta L\right)$ for some $i, 1 \leq i \leq l$. But then

$$
p^{\prime}=\left(\hat{T}^{k-1}\right)^{-1}\left(\operatorname{St}\left(a_{i}, \beta^{2} L\right)\right) \subseteq B
$$

and $\operatorname{Int}\left(p^{\prime} \cap p\right) \neq \varnothing\left(\right.$ by $\left.\left(\mathrm{b}_{k-1}\right)\right)$.

Following the well-established pattern, once again choose a triangulation $K_{k}$ of $S^{m}$ such that all relevant subsets of $S^{m}$ are (full) subcomplexes. Let $\alpha$ be a PL arc in Int $B$ that intersects all sets of the form $p$ or $\operatorname{Int}\left(B \cap p^{\prime}\right)$ for some $p \in \hat{P}^{k-1}$ with $\hat{T}^{k-1}(p) \cap j_{k} \neq \varnothing$ or some $p^{\prime} \in P$ with $T\left(p^{\prime}\right) \cap j_{k} \neq \varnothing$. By Claims 1 and 2 such an arc exists. We can also arrange that it misses the $(m-2)$-skeleton of $K_{k}$. Let $K_{k}^{\prime}$ be a subdivision of $K_{k}$ such that $\alpha$ is a subcomplex, and let $N$ be the second derived neighborhood of $\alpha$ (in $B)$. Define $A(p)=p$-Int $N$ for all $p \in \hat{P}^{k-1}$. Setting $\hat{P}^{k}=\left\{A(p): p \in \hat{P}^{k-1}\right\} \cup\{N\}$ and $\hat{T}^{k}(A(p))=\hat{T}^{k-1}(p), T^{k}(N)=j_{k}$ defines a triple $\hat{T}^{k}: \hat{P}^{k} \rightarrow \tilde{J}$. The reader should observe that this triple satisfies (A2), (A3), (M1), (M2), $\left(\mathrm{a}_{k}\right)$ and $\left(\mathrm{b}_{k}\right)$.

We now proceed with the description of a number of improvements on the construction of a triple $\tilde{T}: \tilde{P} \rightarrow \tilde{J}$. For convenience, we use the following notation. If $p_{i(1)}, \ldots, p_{i(t)} \in P$ with $\bigcap_{r=1}^{t} T\left(p_{i(r)}\right) \neq \varnothing$, then by $A\left(p_{i(1)}, \ldots, p_{i(t)}\right)$ we denote the element of $\tilde{P}$ such that

$$
T\left(A\left(p_{i(1)}, \ldots, p_{i(t)}\right)\right)=\operatorname{St}\left(v, \beta^{2} L\right),
$$

where $v$ is the barycenter of the simplex of $L$ whose vertices are determined by "centers" of $T\left(p_{i(1)}, \ldots, T\left(p_{i(t)}\right)\right)$.

(i) Given $p_{i(1)}, p_{i(2)}, p_{i(3)}, p_{i(4)} \in P$ with $T\left(p_{i(1)}\right) \cap T\left(p_{i(2)}\right) \cap T\left(p_{i(3)}\right) \cap T\left(p_{i(4)}\right)$ $\neq \varnothing$, we can arrange that there exists a PL arc $\alpha \subset \operatorname{Int} p_{i(1)}$ such that $\alpha$ intersects the interior of each of the following elements of $\tilde{P}$, and no other elements of $\tilde{P}: A\left(p_{i(1)}\right), A\left(p_{i(2)}, p_{i(2)}\right), A\left(p_{i(1)}, p_{i(2)}, p_{i(3)}\right), A\left(p_{i(1)}, p_{i(2)}, p_{i(3)}, p_{i(4)}\right)$.

The trick is first to specify an arc $\alpha \subset$ Int $p_{i(1)}$ that meets "right" elements of $\hat{P}$. In the process of "connecting up", we can choose arcs to miss $\alpha$, and (choosing 
a small triangulation of $S^{m}$ ) we can arrange that the "connecting tubes" miss $\alpha$. Consequently, $\alpha$ hits the "right" elements of $\hat{P}^{0}$. In the inductive process of constructing partitions $\hat{P}^{1}, \ldots, \hat{P}^{r}=\tilde{P}$, we can choose the relevant arcs either to hit or to miss $\alpha$ (according to the nature of the partition element that is about to be constructed).

(ii) Along with the hypotheses as in (i), assume that $U$ is an open set in $S^{m}$ and $U \cap p_{i(1)} \neq \varnothing$. Then we can arrange that $\alpha$ (which satisfies the conclusion of (i)) is contained in $U$.

Indeed, if $\alpha$ is any arc as in (i), we can find a PL homeomorphism $\psi: S^{m} \rightarrow S^{m}$ such that $\psi=$ identity off of Int $p_{i(1)}$ and $\psi(\alpha) \subset U$. Then $\tilde{P}^{\prime}=\{\psi(\tilde{p}): \tilde{p} \in \tilde{P}\}$ and $\alpha^{\prime}=\psi(\alpha)$ satisfy all conclusions of (ii).

(iii) Given $p_{i(1)}, p_{i(2)}, p_{i(3)}, p_{i(4)} \in P$ as in (i) and an arc $\alpha \subset \bigcup_{t=1}^{4}$ Int $p_{i(t)}$ intersecting each Int $p_{i(t)}, t=1,2,3,4$, we can arrange that $A\left(p_{i(1)}, p_{i(2)}, p_{i(3)}, p_{i(4)}\right)$ is contained in a prechosen neighborhood $U$ of $\alpha$.

Using an argument of the same type as in (i), we can arrange that $\alpha$ has all properties as an arc serving as a guide for constructing $A\left(p_{i(1)}, p_{i(2)}, p_{i(3)}, p_{i(4)}\right)$. Then it remains to choose a small triangulation of $S^{m}$ to get the required containment (in the inductive process, already "born" elements cannot "grow").

The improvements (ii) and (iii), applied to

$$
A\left(p_{i(1)}\right), A\left(p_{i(1)}, p_{i(2)}\right), A\left(p_{i(1)}, p_{i(2)}, p_{i(3)}\right), A\left(p_{i(1)}, p_{i(2)}, p_{i(3)}, p_{i(4)}\right),
$$

coupled together yield the following (here we use $n \geq 3$ ).

(iv) For any nonempty open set $U \subseteq S^{m}$, in the construction of the defining sequence $\left\{T_{i}: P_{i} \rightarrow J_{i}\right\}_{i=0}^{\infty}$, whenever $P_{i}$ is given, we can arrange (by carefully choosing $P_{i+1}$ and $P_{i+2}$ ) that $P_{i+2}$ contains an element contained in $U$.

(v) Given $p \in P$ and an open set $U \subseteq S^{m}$ with $p \subseteq U$, we can arrange that $\operatorname{St}(A(p), \tilde{P}) \subseteq U$.

Indeed, using (H5), we can arrange that $\operatorname{St}(p, \hat{P}) \subseteq U$. In the "connecting up" process, we choose $c\left(p^{\prime}\right)$ to be $p_{\left\{p^{\prime \prime}\right\}}$ for some $p^{\prime \prime} \in P$. In this way we get $\operatorname{St}\left(\hat{p}, \hat{P}^{0}\right) \subseteq$ $U$ where $\hat{p}=p_{\{p\}}$. Since $\hat{p}$ is connected, we have $\hat{p}^{0} \subseteq \hat{p}$ and hence $\operatorname{St}\left(\hat{p}^{0}, \hat{P}^{0}\right) \subseteq U$. Inductively assume that $\operatorname{St}\left(\hat{p}^{k-1}, \hat{P}^{k-1}\right) \subseteq U$, where $\hat{p} \in \hat{P}^{k-1}$ "comes" from $\hat{p} \in \hat{P}$. If $j_{k} \cap \hat{T}^{k-1}\left(\hat{p}^{k-1}\right)=\varnothing$, we have $\operatorname{St}\left(\hat{p}^{k}, \hat{P}^{k}\right) \subseteq \operatorname{St}\left(\hat{p}^{k-1}, \hat{P}^{k-1}\right) \subseteq U$. So assume that $j_{k} \cap \hat{T}^{k-1}\left(\hat{p}^{k-1}\right) \neq \varnothing$. The corresponding set $B$ defined along the inductive argument can be written as $B=B_{1} \cup B_{2}$, where $B_{1}=\bigcup\left\{p^{\prime} \in \hat{P}^{k-1}: p^{\prime} \subseteq B, p^{\prime} \cap\right.$ $\left.\hat{p}^{k-1} \neq \varnothing\right\}$, and $B_{2}=\bigcup\left\{p^{\prime} \in \hat{P}^{k-1}: p^{\prime} \cap \hat{p}^{k-1}=\varnothing\right\}$. Then $B_{1} \subseteq U$ and each $p^{\prime} \subseteq B_{2}$ hits $B_{1}$. Consequently, if we replace the set $B$ in the inductive argument by the set $B^{\prime}=B_{1} \cup\left\{\right.$ the collection of all components of $B_{2} \cap U$ that hit $\left.B_{1}\right\}$, the constructed element $N$ will be contained in $U$, and hence $\operatorname{St}\left(\hat{p}^{k}, \hat{P}^{k}\right) \subseteq U$.

Improvements (iv) and (v) give the following:

Let $\left\{U_{1}, U_{2}, U_{3}, \ldots\right\}$ be a countable basis of open sets for the topology on $S^{m}$. Then we can arrange that, for each $i$, there exists $p(i) \in P_{3 i}$ with $\operatorname{St}\left(p(i), P_{3 i}\right) \subseteq U_{i}$.

Since by 2.1(i) each of the sets of the form $\operatorname{St}(p, P), p \in P$, contains a fiber of the map $h: S^{m} \rightarrow S^{n}$ determined by such a defining sequence, we conclude that $h$ satisfies the hypotheses of 4.2 , and hence it is one-to-one over the image of a dense set. (M1) implies that $h$ is monotone. 


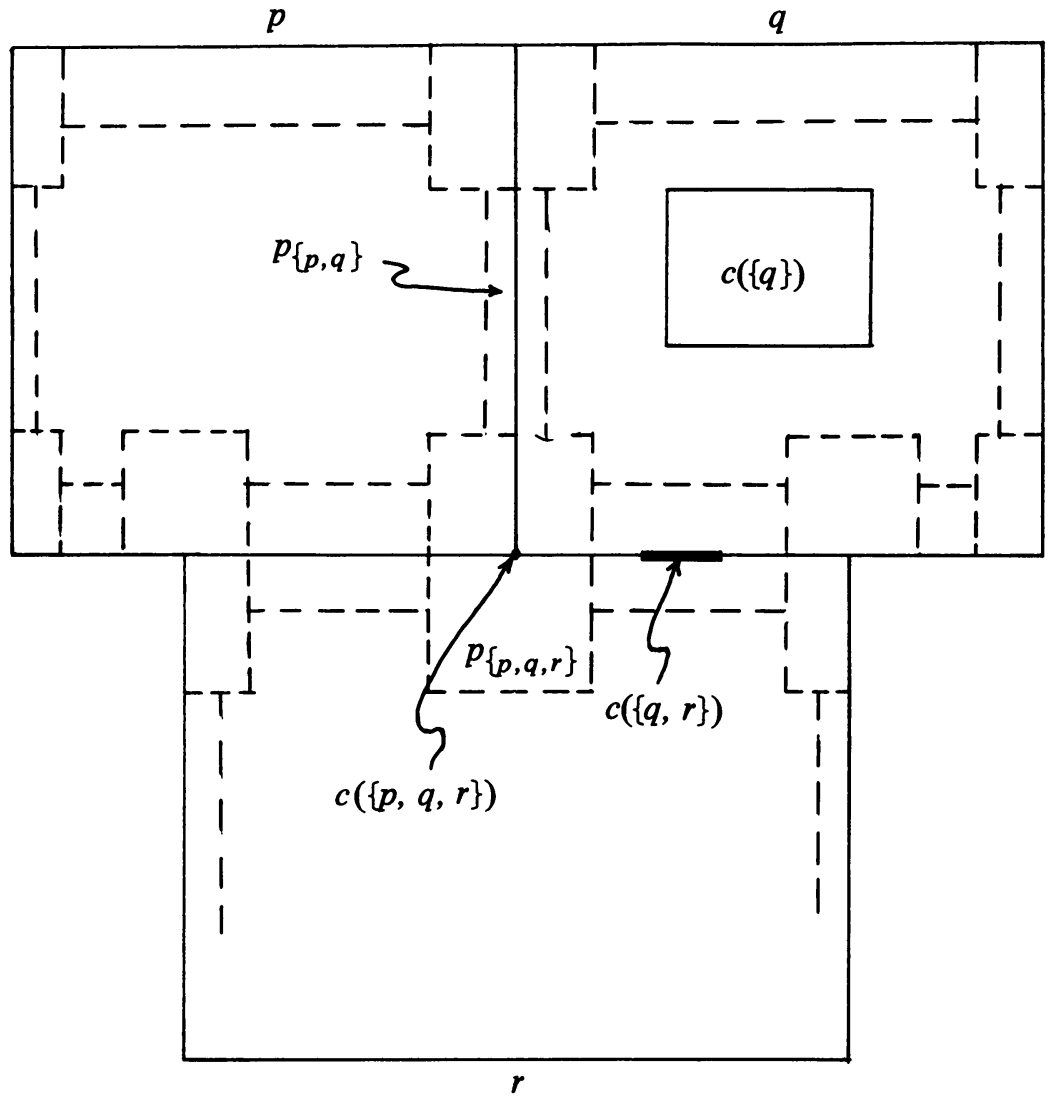

Figure 1

To finish the proof of 4.1, we need to construct a triple $T_{0}: P_{0} \rightarrow J_{0}$ satisfying (A1)-(A3) and (M1), (M2). As in $\S 3$, we take advantage of the fact that spheres are suspensions.

Let $L^{\prime}$ be any triangulation of $S^{n-1}$. Then there exists a partition $P$ of $S^{m-1}$ and an admissible map $T: P \rightarrow J, J=\left\{\operatorname{St}\left(v, \beta L^{\prime}\right): v\right.$ is a vertex of $\left.L^{\prime}\right\}$ such that if $p_{i(1)}, p_{i(2)}, p_{i(3)} \in P$ are distinct, then $p_{i(1)} \cap p_{i(2)} \cap p_{i(3)}=\varnothing$. We now "suspend" the triple $T: P \rightarrow J$. Let $J_{0}$ be the standard handlebody decomposition of $S^{n}$ corresponding to the triangulation $L=\Sigma L^{\prime} . P$ is a partition of $S^{m-1} \subset S^{m}$. Let $P_{0}^{\prime}$ be the partition of $S^{m}$ consisting of slightly "thickened" copies of $p \in P$ together with two $m$-balls corresponding to the suspension points. Defining $T_{0}^{\prime}: P_{0}^{\prime} \rightarrow J_{0}$ in the obvious way, the reader should realize that this triple satisfies (A1)-(A3) and (M2). It remains to "connect up" elements of $P_{0}^{\prime}$. Observing that both $m$ balls in $P_{0}^{\prime}$, corresponding to two suspension points, intersect all components of all disconnected elements of $P_{0}^{\prime}$, the author leaves this as an exercise.

This completes the proof of 4.1.

REMARK 4.3. In [Be-Wa] it is shown that a map $f: S^{m} \rightarrow S^{2}$ that is one-toone over the image of a dense set is far from being monotone. Using the technique of this section, one can construct a map $f: S^{m} \rightarrow S^{2}$ that is one-to-one over the image of a dense set, thus giving an alternative proof of the result in $[\mathbf{B e}-\mathbf{W a}]$. One 
finds a defining sequence $\left\{T_{i}: P_{i} \rightarrow J_{i}\right\}_{i=0}^{\infty}$ with the additional property:

(U) If $p_{i(1)}, p_{i(2)}, p_{i(3)} \in P$ are mutually distinct, then $p_{i(1)} \cap p_{i(2)} \cap p_{i(3)}=\varnothing$.

The argument is slightly easier than the one given in the case of monotone maps, since one does not have to worry about "connecting up" various components.

REMARK 4.4. Maps $h: S^{m} \rightarrow S^{n}$ constructed in 4.1 and 4.3, are totally unstable (i.e. they have no stable values). In fact $h: S^{m} \rightarrow S^{n}$ can be approximated by a map $h_{i}: S^{m} \rightarrow S^{n}$ induced by the admissible function $T_{i}: P_{i} \rightarrow J_{i}$, which, in turn, can be approximated by a map $h_{i}^{\prime}: S^{m} \rightarrow S^{n}$ induced by the triple $\hat{T}_{i}: \hat{P}_{i} \rightarrow \tilde{J}_{i}$. If $T_{i}: P_{i} \rightarrow J_{i}$ satisfies (M2), then $\operatorname{Im} h_{i}^{\prime}$ is contained in the union of all elements $j$ of $J_{i}$ with $\operatorname{Ind}(j) \leq 2$, and hence it is contained in a regular neighborhood of the 2-skeleton of $\beta^{i} L$. Consequently, $h: S^{m} \rightarrow S^{n}$ constructed as in 4.1 can be approximated by maps that factor through 2-dimensional polyhedra.

\section{REFERENCES}

[Be-Wa] M. Bestvina and J. J. Walsh, Mappings between Euclidean spaces that are one to one over the image of a dense subset, Proc. Amer. Math. Soc. 91 (1984), 449-455.

[Du] J. Dugundji, Topology, Allyn \& Bacon, Boston, Mass., 1966.

[Sp] E. H. Spanier, Algebraic Topology, McGraw-Hill, New York, 1966.

[Wa 1 ] J. J. Walsh, Monotone and open mappings on manifolds. I, Trans. Amer. Math. Soc. 209 (1975), 419-432.

[Wa 2] _ Light open and open mappings on manifolds. II, Trans. Amer. Math. Soc. 217 (1976), 271-284.

[Wa 3] _ Isotoping mappings to open mappings, Trans. Amer. Math. Soc. 250 (1979), 121-145.

[Wa 4] _ Extending mappings to monotone mappings, Houston J. Math. 3 (1977), 579-592.

[Wa 5] _ Mappings with dense deficiency set, Michigan Math. J. 28 (1981), 117-127.

[Wi 1] D. C. Wilson, Open mappings on manifolds and a counterexample to the Whyburn conjecture, Duke Math. J. 40 (1973), 705-716.

[Wi 2] _ Monotone mappings of manifolds onto cells, Proc. First Conf. Monotone and Open Mappings (SUNY, Binghamton, 1970), SUNY, Binghamton, New York, 1971, pp. 37-54.

Department of Mathematics, University of Tennessee, Knoxville, TenNESSEE 37996-1300

Current address: Mathematical Sciences Research Institute, 2223 Fulton Street, Berkeley, California, 94720 